\title{
Expansions Involving Hypergeometric Functions of Two Variables
}

\section{By Arun Verma}

1. Introduction. A systematic study of expansions of Appell's hypergeometric functions of two variables has been made by Burchnall and Chaundy [3]-[5] in 1942. Recently, Ragab [11], obtained two expansions of Kampé de Fériet's double hypergeometric functions which besides incorporating some of the expansions of Burchnall and Chaundy [3], [4], gave some new expansions also.

In $\S 2$ of this paper a systematic attempt has been made to extend the expansions concerning Appell functions to the Kampé de Fériet's double hypergeometric functions by using the two symbolic operators of Burchnall and Chaundy. In $\$ 4$ using the method of iteration of series some of the expansions due to Chaundy [:], [6], Niblett [10], Wimp and Luke [8] have been extended to the Kampé de Fériet's function. The paper is concluded by showing how the induction by using the Laplace transform and its inverse can be employed to extend these results to $G$-functions of two variables defined recently by Agarwal [1] ${ }^{*}$. It may be pointed out that these expansions are very general in nature and incorporate a very large number of expansions for the functions of two variables.

2. Kampé de Fériet† [2] introduced the double hypergeometric functions of higher order (i.e. with more parameters) in two variables, namely:

$$
F\left[\begin{array}{l|l|r}
\mu & \left(\alpha_{\mu}\right) & \\
\nu & \left(\beta_{\nu}\right) ;\left(\beta_{\nu}{ }^{\prime}\right) & x \\
\rho & \left(\gamma_{\rho}\right) & y \\
\sigma & \left(\delta_{\sigma}\right) ;\left(\delta_{\sigma}{ }^{\prime}\right) &
\end{array}\right]=\sum_{m=0}^{\infty} \sum_{n=0}^{\infty} \frac{\left[\left(\alpha_{\mu}\right)\right]_{m+n}\left[\left(\beta_{\nu}\right)\right]_{m}\left[\left(\beta_{\nu}{ }^{\prime}\right)\right]_{n} x^{m} y^{n}}{[1]_{n}\left[\left(\gamma_{\rho}\right)\right]_{m+n}\left[\left(\delta_{\sigma}\right)\right]_{m}\left[\left(\delta_{\sigma}{ }^{\prime}\right)\right]_{n}},
$$

provided $\mu+\nu<\rho+\sigma+1$ or $\mu+\nu=\rho+\sigma+1$ and $|x|+|y|<$ $\min \left(1,2^{\rho-\mu+1}\right)$.

Here $\left(a_{A}\right)$ means $A$ parameters of the type $a_{1}, a_{2}, \cdots, a_{A}$ and

$$
[a]_{n}=a[a+1][a+2] \cdots[a+n-1] ; \quad[a]_{0}=1 .
$$

For special values of $\mu, \nu, \rho, \sigma$ the function (1) reduces to the Appell's four double hypergeometric functions. Following Burchnall and Chaundy [3], [4], we define the operators $\Delta(h)$ and $\nabla(h)$ as

$$
\Delta(h)=\Gamma\left[\begin{array}{l}
\delta+h, \delta^{\prime}+h ; \\
h, \delta+\delta^{\prime}+h
\end{array}\right], \quad \nabla(h)=\Gamma\left[\begin{array}{l}
h, \delta+\delta^{\prime}+h ; \\
\delta+h, \delta^{\prime}+h
\end{array}\right],
$$

where $\delta \equiv x \partial / \partial x, \delta^{\prime} \equiv y \partial / \partial y$.

Now it can be seen without difficulty

Received February 21, 1966.

* I am indebted to Dr. R. P. Agarwal for letting me read his unpublished manuscript [1].

$\dagger$ For definition and properties of these functions, see [2]. 


$$
\begin{aligned}
& \nabla(a) F\left[\begin{array}{l|l|l}
\mu & \left(\alpha_{\mu}\right) & \\
\nu & \left(\beta_{\nu}\right) ;\left(\beta_{\nu}{ }^{\prime}\right) & x \\
\rho & \left(\gamma_{\rho}\right) & y \\
\sigma & \left(\delta_{\sigma}\right) ;\left(\delta_{\sigma}{ }^{\prime}\right) &
\end{array}\right]=F\left[\begin{array}{c|l|l}
\mu+1 & \left(\alpha_{\mu}\right), a \\
\nu & \left(\beta_{\nu}\right) ;\left(\beta_{\nu}{ }^{\prime}\right) & x \\
\rho & \left(\gamma_{\rho}\right) & y \\
\sigma+1 & \left(\delta_{\sigma}\right), a ;\left(\delta_{\sigma}{ }^{\prime}\right), a & y
\end{array}\right], \\
& \Delta(a) F\left[\begin{array}{l|l|l}
\mu & \left(\alpha_{\mu}\right) \\
\nu & \left(\beta_{\nu}\right) ;\left(\beta_{\nu}{ }^{\prime}\right) & x \\
\rho & \left(\gamma_{\rho}\right) & y \\
\sigma & \left(\delta_{\sigma}\right) ;\left(\delta_{\sigma}{ }^{\prime}\right) & y
\end{array}\right]=F\left[\begin{array}{l|l|l}
\mu & \left(\alpha_{\mu}\right) \\
\nu+1 & \left(\beta_{\nu}\right), a ;\left(\beta_{\nu}{ }^{\prime}\right), a & x \\
\rho+1 & \left(\gamma_{\rho}\right), a \\
\sigma & \left(\delta_{\sigma}\right) ;\left(\delta_{\sigma}{ }^{\prime}\right)
\end{array}\right], \\
& \nabla(a) \Delta(c) F\left[\begin{array}{l|l|l}
\mu & \left(\alpha_{\mu}\right) & \\
\nu & \left(\beta_{\nu}\right) ;\left(\beta_{\nu}{ }^{\prime}\right) & x \\
\rho & \left(\gamma_{\rho}\right) & y \\
\sigma & \left(\delta_{\sigma}\right) ;\left(\delta_{\sigma}{ }^{\prime}\right) &
\end{array}\right]=F\left[\begin{array}{l|l|l}
\mu+1 & \left(\alpha_{\mu}\right), a \\
\nu+1 & \left(\beta_{\nu}\right), c ;\left(\beta_{\nu}{ }^{\prime}\right), c & x \\
\rho+1 & \left(\gamma_{\rho}\right), c \\
\sigma+1 & \left(\delta_{o}\right), a ;\left(\delta_{\sigma}{ }^{\prime}\right), a & y
\end{array}\right] .
\end{aligned}
$$

Then following the method of Burchnall and Chaundy and using the following identities

$$
\begin{aligned}
& \Gamma\left[\begin{array}{l}
h, m+n+h ; \\
m+h, n+h
\end{array}\right]=\sum_{r=0}^{\infty} \frac{[-m]_{r}[-n]_{r}}{[1]_{r}[h]_{r}} \\
& \Gamma\left[\begin{array}{l}
m+h, n+h ; \\
h, m+n+h
\end{array}\right]=\sum_{r=0}^{\infty} \frac{[-m]_{r}[-n]_{r}}{[1]_{r}[-h-m-n+1]_{r}} \\
& =\sum_{r=0}^{\infty} \frac{[-]^{r}[h]_{2 r}[-m]_{r}[-n]_{r}}{[1]_{r}[h+r-1]_{r}[m+h]_{r}[n+h]_{r}} \\
& =\sum_{r=0}^{\infty} \frac{[k-h]_{r}[k]_{2 r}[-m]_{r}[-n]_{r}}{[1]_{r}[k+r-1]_{r}[m+k]_{r}[n+k]_{r}[h]_{r}} \\
& \text { (limiting form of Dougall's theorem) } \\
& =\sum_{r=0}^{\infty} \frac{[h-k]_{r}[-m]_{r}[-n]_{r}}{[1]_{r}[h]_{r}[-k-m-n+1]_{r}}
\end{aligned}
$$

(Saalschütz's theorem with either $m$ or $n$ a positive integer) we get the following five general expansions

$$
\begin{aligned}
(10) \quad F\left[\begin{array}{l|l|l}
\mu & \left(\alpha_{\mu}\right) \\
\nu & \left(\beta_{\nu}\right) ;\left(\beta_{\nu}{ }^{\prime}\right) & x \\
\rho & \left(\gamma_{\rho}\right) & x \\
\sigma & \left(\delta_{\sigma}\right) ;\left(\delta_{\sigma}{ }^{\prime}\right) & y
\end{array}\right] & =\sum_{r=0}^{\infty} \frac{\left[\left(\alpha_{\mu}\right)\right]_{2 r}\left[\left(\beta_{\nu}\right)\right]_{r}[a]_{r}\left[\left(\beta_{\nu}{ }^{\prime}\right)\right]_{r}}{[1]_{r}\left[\left(\gamma_{\rho}\right)\right]_{2 r}[a]_{2 r}\left[\left(\delta_{\sigma}\right)\right]_{r}\left[\left(\delta_{\sigma}{ }^{\prime}\right)\right]_{r}} x^{r} y^{r} \\
\times F & {\left[\begin{array}{l|l|l}
\mu & \left(\alpha_{\mu}\right)+2 r & \begin{array}{l}
a+r,\left(\beta_{\nu}\right)+r ; a+r,\left(\beta_{\nu}{ }^{\prime}\right)+r \\
\nu+1
\end{array} \\
\rho+1 & \begin{array}{l}
a+2 r,\left(\gamma_{\rho}\right)+2 r \\
\left(\delta_{\sigma}\right)+r ;\left(\delta_{\sigma}{ }^{\prime}\right)+r
\end{array}
\end{array}\right] . }
\end{aligned}
$$




$$
\begin{gathered}
=\sum_{r=0}^{\infty} \frac{[-]^{r}\left[\left(\alpha_{\mu}\right)\right]_{2 r}\left[\left(\beta_{\nu}\right)\right]_{r}\left[\left(\beta_{\nu}{ }^{\prime}\right)\right]_{r}}{[1]_{r}\left[\left(\gamma_{\rho}\right)\right]_{2 r}\left[\left(\delta_{\sigma}\right)\right]_{r}\left[\left(\delta_{\sigma}{ }^{\prime}\right)\right]_{r}[a]_{r}} x^{r} y^{r} \\
\left.\qquad \begin{array}{c|l|l}
1+\mu & \left(\alpha_{\mu}\right)+2 r, a+r \\
\nu & \left(\beta_{\nu}\right)+r ;\left(\beta_{\nu}{ }^{\prime}\right)+r & x \\
\rho & \left(\gamma_{\rho}\right)+2 r & y \\
1+\sigma & \left(\delta_{\sigma}\right)+r, a+r ;\left(\delta_{\sigma}{ }^{\prime}\right)+r, a+r & y
\end{array}\right] .
\end{gathered}
$$

$$
=\sum_{r=0}^{\infty} \frac{[c-a]_{r}\left[\left(\alpha_{\mu}\right)\right]_{2 r}\left[\left(\beta_{\nu}\right)\right]_{r}\left[\left(\beta_{\nu}{ }^{\prime}\right)\right]_{r}[a]_{r}}{[1]_{r}[c+r-1]_{r}\left[\left(\gamma_{\rho}\right)\right]_{2 r}[a]_{2 r}\left[\left(\delta_{\sigma}\right)\right]_{r}\left[\left(\delta_{\sigma}{ }^{\prime}\right)\right]_{r}} x^{r} y^{r}
$$

$$
\times F\left[\begin{array}{l|l|l}
\mu+1 & \left(\alpha_{\mu}\right)+2 r, c+2 r & \\
\nu+1 & \left(\beta_{\nu}\right)+r, a+r ;\left(\beta_{\nu}{ }^{\prime}\right)+r, a+r & x \\
\rho+1 & \left(\gamma_{\rho}\right)+2 r, a+2 r & y \\
\sigma+1 & \left(\delta_{\sigma}\right)+r, c+2 r ;\left(\delta_{\sigma}{ }^{\prime}\right)+r, c+2 r &
\end{array}\right] .
$$

$$
\begin{gathered}
=\sum_{r=0}^{\infty} \frac{(-)^{r}\left[\left(\alpha_{\mu}\right)\right]_{2 r}\left[\left(\beta_{\nu}\right)\right]_{r}\left[\left(\beta_{\nu}{ }^{\prime}\right)\right]_{r}}{[1]_{r}[a+r-1]_{r}\left[\left(\gamma_{\rho}\right)\right]_{2 r}\left[\left(\delta_{\sigma}\right)\right]_{r}\left[\left(\delta_{\sigma}{ }^{\prime}\right)\right]_{r}} x^{r} y^{r} \\
\left.\qquad \begin{array}{c|l|l}
\mu+1 & \left(\alpha_{\mu}\right)+2 r, a+r & \\
\nu & \left(\beta_{\nu}\right)+r ;\left(\beta_{\nu}{ }^{\prime}\right)+r & x \\
\rho & \left(\gamma_{\rho}\right)+2 r & y \\
\sigma+1 & \left(\delta_{\sigma}\right)+r, a+2 r ;\left(\delta_{\sigma}{ }^{\prime}\right)+r, a+2 r
\end{array}\right] .
\end{gathered}
$$

$$
=\sum_{r=0}^{\infty} \frac{(-)^{r}[a-c]_{r}\left[\left(\alpha_{\mu}\right)\right]_{2 r}\left[\left(\beta_{\nu}\right)\right]_{r}\left[\left(\beta_{\nu}{ }^{\prime}\right)\right]_{r}[a]_{r}}{[1]_{r}\left[\left(\gamma_{\rho}\right)\right]_{2 r}[a]_{2 r}\left[\left(\delta_{\sigma}\right)\right]_{r}\left[\left(\delta_{\sigma}{ }^{\prime}\right)\right]_{r}} x^{r}
$$

$$
\times F\left[\begin{array}{l|l|l}
\mu+1 & \left(\alpha_{\mu}\right)+2 r, c+r & \\
\nu+1 & \left(\beta_{\nu}\right)+r, a+r ;\left(\beta_{\nu}{ }^{\prime}\right)+r, a+r & x \\
\rho+1 & \left(\gamma_{\rho}\right)+2 r, a+2 r & y \\
\sigma+1 & \left(\delta_{\sigma}\right)+r, c+r ;\left(\delta_{\sigma}{ }^{\prime}\right)+r, c+r &
\end{array}\right] .
$$

It may be pointed out that the Eq. (10) on specialization of parameters gives Eqs. (26), (29), (32), (35), (36), (38), (41) and our (11) gives (27), (33), (37), (39); (12) gives (30) and (42); (13) gives Equations (28), (34) and (40); (14) gives Eqs. (31) and (41) of Burchnall and Chaundy [3]. The above expansions (12) and (13) were deduced by Ragab [11] by the iteration of series.

3. In this section, we first state and then prove some more general expansions

$$
\begin{aligned}
& F\left[\begin{array}{c|l|r}
A+G & \left(a_{A}\right),\left(g_{G}\right) \\
B+H & \left(b_{B}\right),\left(h_{H}\right) ;\left(b_{B}{ }^{\prime}\right),\left(h_{H}{ }^{\prime}\right) & \lambda x \\
C & \left(c_{C}\right) & \\
D+E & \left(d_{D}\right),\left(e_{E}\right) ;\left(d_{D}{ }^{\prime}\right),\left(e_{E}{ }^{\prime}\right) & \mu y
\end{array}\right] \\
& =h h^{\prime} \sum_{m=0}^{\infty} \sum_{n=0}^{\infty} \frac{\left[\left(f_{\boldsymbol{F}}\right)\right]_{m+n}\left[\left(g_{G}\right)\right]_{m+n}\left[\left(h_{H}\right)\right]_{m}\left[\left(h_{H}{ }^{\prime}\right)\right]_{n}[h+\alpha m-m+1]_{m-1}}{[1]_{m}[1]_{n}\left[\left(c_{C}\right)\right]_{m+n}\left[\left(d_{D}\right)\right]_{m}\left[\left(d_{D}{ }^{\prime}\right)\right]_{n}} \\
& \times\left[h^{\prime}+\beta n-n+1\right]_{n-1} \\
& \times F\left[\begin{array}{c|l|r}
A & \left(a_{A}\right) & \\
B+2 & \left(b_{B}\right), 1+h / \alpha,-m ;\left(b_{B}{ }^{\prime}\right), 1+h^{\prime} / \beta,-n & \lambda \\
F & \left(f_{F}\right) & \mu \\
E+2 & \left(e_{B}\right), h+\alpha m-m+1, h / \alpha ;\left(e_{E}{ }^{\prime}\right), h^{\prime}+\beta n-n+1, h / \beta &
\end{array}\right] \\
& \times(-x)^{m}(-y)^{n} F\left[\begin{array}{c|l|r}
F+G & \left(f_{F}\right)+m+n,\left(g_{G}\right)+m+n \\
2+H & \left(h_{H}\right)+m, h+\alpha m ;\left(h_{H}{ }^{\prime}\right)+n, h^{\prime}+\beta n \\
C & \left(c_{C}\right)+m+n \\
D & \left(d_{D}\right)+m ;\left(d_{D}{ }^{\prime}\right)+n
\end{array}\right] y
\end{aligned}
$$




$$
\begin{aligned}
F\left[\begin{array}{l|l|l}
A & \left(a_{A}\right) & \\
B & \left(b_{B}\right) ;\left(b_{B}{ }^{\prime}\right) & \lambda x \\
C & \left(c_{C}\right) & \mu y \\
D & \left(d_{D}\right) ;\left(d_{D}{ }^{\prime}\right) &
\end{array}\right] \\
=\sum_{r=0}^{\infty} \sum_{s=0}^{\infty} \frac{(-)^{r+s}\left[\left(\alpha_{\mu}\right)\right]_{r+s}\left[\left(\beta_{\nu}\right)\right]_{r}\left[\left(\beta_{\nu}{ }^{\prime}\right)\right]_{s}}{[1]_{r}[1]_{s}\left[\left(\gamma_{\rho}\right)\right]_{r+s}\left[\left(\delta_{\sigma}\right)\right]_{r}\left[\left(\delta_{\sigma}{ }^{\prime}\right)\right]_{s}}
\end{aligned}
$$

$$
\begin{aligned}
& \times x^{r} y^{s} F\left[\begin{array}{l|l|r}
A+\rho & \left(a_{A}\right),\left(\gamma_{\rho}\right) & \\
B+\sigma+1 & \left(b_{B}\right),\left(\delta_{\sigma}\right),-r ;\left(b_{B}{ }^{\prime}\right),\left(\delta_{\sigma}{ }^{\prime}\right),-s & \lambda \\
C+\mu & \left(c_{C}\right),\left(\alpha_{\mu}\right) & \mu \\
D+\nu & \left(d_{D}\right),\left(\beta_{\nu}\right) ;\left(d_{D}{ }^{\prime}\right),\left(\beta_{\nu}{ }^{\prime}\right) & \mu
\end{array}\right] \\
& \times F\left[\begin{array}{c|l|l}
\mu & \left(\alpha_{\mu}\right)+r+s & \\
\nu & \left(\beta_{\nu}\right)+r ;\left(\beta_{\nu}{ }^{\prime}\right)+s & x \\
\rho & \left(\gamma_{\rho}\right)+r+s & y \\
\sigma & \left(\delta_{\sigma}\right)+r ;\left(\delta_{\sigma}{ }^{\prime}\right)+s &
\end{array}\right] \text {. } \\
& F\left[\begin{array}{l|l|r}
A & \left(a_{A}\right) \\
B & \left(b_{B}\right) ;\left(b_{B}{ }^{\prime}\right) & \lambda x \\
C & \left(c_{C}\right) & \mu y \\
D & \left(d_{D}\right) ;\left(d_{D}{ }^{\prime}\right) &
\end{array}\right]
\end{aligned}
$$$$
=\sum_{r=0}^{\infty} \sum_{s=0}^{\infty} \frac{(-)^{r+s}\left[\left(\alpha_{\mu}\right)\right]_{r+s}\left[\left(\beta_{\nu}\right)\right]_{r}\left[\left(\beta_{\nu}{ }^{\prime}\right)\right]_{s}}{[1]_{r}[1]_{s}\left[\left(\gamma_{\rho}\right)\right]_{r+s}\left[\left(\delta_{\sigma}\right)\right]_{r}\left[\left(\delta_{\sigma}{ }^{\prime}\right)\right]_{s}}[c+r+s-1]_{r+s} x^{r} y^{s}
$$$$
\times F\left[\begin{array}{l|l|l}
1+A+\rho & c+r+s-1,\left(a_{A}\right),\left(\gamma_{\rho}\right) & \\
1+B+\sigma & -r,\left(b_{B}\right),\left(\delta_{\sigma}\right) ;-s,\left(b_{B}{ }^{\prime}\right),\left(\delta_{\sigma}{ }^{\prime}\right) & \lambda \\
C+\mu & \left(c_{C}\right),\left(\alpha_{\mu}\right) & \mu \\
D+\nu & \left(d_{D}\right),\left(\beta_{\nu}\right) ;\left(d_{D}{ }^{\prime}\right),\left(\beta_{\nu}{ }^{\prime}\right) &
\end{array}\right]
$$

$$
\begin{aligned}
& \times F\left[\begin{array}{l|l|l}
\mu & \left(\alpha_{\mu}\right)+r+s & \\
\nu & \left(\beta_{\nu}\right)+r ;\left(\beta_{\nu}{ }^{\prime}\right)+s & x \\
\rho+1 & c+2 r+2 s,\left(\gamma_{\rho}\right)+r+s & y \\
\sigma & \left(\delta_{\sigma}\right)+r ;\left(\delta_{\sigma}^{\prime}\right)+s
\end{array}\right] . \\
& F\left[\begin{array}{l|l|l}
p & \left(A_{p}\right) & \\
q & \left(B_{q}\right) ;\left(B_{q}{ }^{\prime}\right) & \lambda x \\
E+D & \left(e_{E}\right),\left(d_{D}\right) & \mu y \\
u+F & \left(c_{u}\right),\left(f_{F}\right) ;\left(c_{u}{ }^{\prime}\right),\left({f_{F}}^{\prime}\right) &
\end{array}\right] \\
& =\sum_{r \rightarrow 0}^{\infty} \sum_{s=0}^{\infty} \frac{(-)^{r+s}\left[\left(a_{w}\right)\right]_{r+s}\left[\left(b_{v}\right)\right]}{[1]_{r}[1]_{s}[c+r-1]_{r}} r
\end{aligned}
$$

$$
\times \frac{\left[\left(b_{v}^{\prime}\right)\right]_{s} x^{r} y^{s}}{\left[c^{\prime}+s-1\right]_{s}\left[\left(e_{E}\right)\right]_{r+s}\left[\left(f_{F}\right)\right]_{r}\left[\left(f_{F}^{\prime}\right)\right]_{s}}
$$$$
\times F\left[\begin{array}{l|l|l}
w & \left(a_{w}\right)+r+s & \\
v & \left(b_{v}\right)+r ;\left(b_{v}\right)+s & x \\
E & \left(e_{E}\right)+r+s & y \\
1+F & c+2 r,\left(f_{F}\right)+r ; c^{\prime}+2 s,\left(f_{F}\right) &
\end{array}\right]
$$$$
\times F\left[\begin{array}{l|l|l}
p & \left(A_{p}\right) \\
2+q & c+r-1,-r,\left(B_{q}\right) ; c^{\prime}+s-1,-s,\left(B_{q}{ }^{\prime}\right) & \lambda \\
w+D & \left(a_{w}\right),\left(d_{D}\right) & \mu \\
u+v & \left(c_{u}\right),\left(b_{v}\right) ;\left(c_{u}{ }^{\prime}\right),\left(b_{v}{ }^{\prime}\right) &
\end{array}\right] .
$$ 
To prove (15) equate the coefficients of

$$
\frac{\left[\left(a_{A}\right)\right]_{M+N}\left[\left(b_{B}\right)\right]_{M}\left[\left(b_{B}{ }^{\prime}\right)\right]_{N}}{[1]_{M}[1]_{N}\left[\left(e_{E}\right)\right]_{M}\left[\left(e_{E}{ }^{\prime}\right)\right]_{N}} \lambda^{M} \mu^{N}
$$

on both the sides and then put $M+r=m, N+s=n$ after writing the series definition for the Kampé de Fériet's function. We then get

$$
\begin{aligned}
& 1=[h+\alpha M]\left[h^{\prime}+\beta N\right] \\
& \times \sum_{r=0}^{\infty} \sum_{s=0}^{\infty} \sum_{P=0}^{\infty} \sum_{Q=0}^{\infty} \frac{(-)^{r+s}\left[\left(f_{F}\right)+M+N\right]_{P+Q+r+s}\left[\left(g_{G}\right)+M+N\right]_{P+Q+r+s}}{[1]_{P}[1]_{Q}[1]_{r}[1]_{s}\left[\left(c_{C}\right)+M+N\right]_{P+Q+r+s}} \\
& {\left[\left(h_{H}\right)+M\right]_{r+P}\left[\left(h_{H}{ }^{\prime}\right)+N\right]_{s+Q}[1+h+r(\alpha-1)} \\
& \times \frac{+M \alpha]_{r+P-1}\left[1+h^{\prime}+s(\beta-1)+N \beta\right]_{s+Q-1}}{\left[\left(d_{D}\right)+M\right]_{r+P}\left[\left(d_{D}{ }^{\prime}\right)+N\right]_{s+Q}} .
\end{aligned}
$$

Next, set $r+P=T, s+Q=U$ and change the order of summation. Then it is not difficult to see that the coefficient of the term independent of $x y$ on the right-hand side is unity. It thus remains to show that the coefficient of $x^{T} y^{U}$ on the right-hand side $(T>0, U>0)$ vanish, i.e.

$0=\sum_{r=0}^{T} \sum_{s=0}^{U} \frac{(-)^{r+s}[1+h+r(\alpha-1)+M \alpha]_{T-1}\left[1+h^{\prime}+s(\beta-1)+N \beta\right]_{U-1}}{[1]_{r}[1]_{s}[1]_{T-r}[1]_{U-s}}$,

which is a known result due to Chaundy [6]. It may be remarked that this result is a generalisation of a result due to Chaundy [6] and contains as a special case a result due to Niblett [10].

To prove (16), equate the coefficients of

$$
\frac{\left[\left(a_{A}\right)\right]_{M+N}\left[\left(b_{B}\right)\right]_{M}\left[\left(b_{B}{ }^{\prime}\right)\right]_{N}}{[1]_{M}[1]_{N}\left[\left(c_{C}\right)\right]_{M+N}\left[\left(d_{D}\right)\right]_{M}\left[\left(d_{D}\right)\right]_{N}}, \lambda^{M} \mu^{N}
$$

on both the sides and then using the series definition of the Kampé de Fériet's function and setting $r+m=P$ and $s+n=Q$, we get that

$$
\begin{aligned}
x^{M I} y^{N}=\frac{\left[\left(\gamma_{\rho}\right)\right]_{M+N}\left[\left(\delta_{\sigma}\right)\right]_{M}\left[\left(\delta_{\sigma}{ }^{\prime}\right)\right]_{N}}{\left[\left(a_{\mu}\right)\right]_{M+N}\left[\left(\beta_{\nu}\right)\right]_{M}\left[\left(\beta_{\nu}{ }^{\prime}\right)\right]_{N}} \sum_{P=0}^{\infty} \sum_{Q=0}^{\infty} \frac{\left[\left(\alpha_{\mu}\right)\right]_{P+Q}\left[\left(\beta_{\nu}\right)\right]_{P}\left[\left(\beta_{\nu}{ }^{\prime}\right)\right]_{Q}}{\left[\left(\gamma_{\rho}\right)\right]_{P+Q}\left[\left(\delta_{\sigma}\right)\right]_{P}\left[\left(\delta_{\sigma}\right)\right]_{Q}} x^{Q} y^{Q} \\
\quad \times\left\{\sum_{r=0}^{P} \sum_{s=0}^{Q} \frac{(-)^{r+s+M+N}}{[1]_{r-M}[1]_{s-N}[1]_{P-r}[1]_{Q-s}}\right\} .
\end{aligned}
$$

Using a lemma due to Chaundy, we find that for $P=M ; s=Q$ the double series in the crooked brackets is equal to 1 and for $P>s, Q>N$ its value is zero and hence the result is established.

This result incorporates as special case some of the results due to Chaundy (Eqs. (8), (9), (10) and (11) of [5]) and for $y=\mu=0$ this reduces to a result due to Jerry L. Fields and Jet Wimp which in turn contains a large number of other expansions as special cases.

To prove (17), equate the coefficient of

$$
\frac{\left[\left(a_{A}\right)\right]_{M+N}\left[\left(b_{B}\right)\right]_{M}\left[\left(b_{B}\right)\right]_{N}^{M_{N}}}{[1]_{M}[1]_{N}\left[\left(c_{C}\right)\right]_{M+N}\left[\left(d_{D}\right)\right]_{M}\left[\left(d_{D}^{\prime}\right)\right]_{N}}
$$


on both the sides, then use the series definition of the Kampé de Fériet's function and use the second lemma of Chaundy [5], after setting $P+r=m$ and $Q+s=n$ to get the desired result.

This result also contains as a special case some of the expansions due to Chaundy [5] and reduces for $y=\mu=0$ to a result due to Jerry L. Fields and Jet Wimp [7].

The proof of (18) can be developed on exactly similar lines. This result also gives as special cases some known expansions due to Fields and Wimp.

4. In this section we shall extend the results deduced in the previous sections to $G$-functions of two variables. Agarwal [1] defined a $G$-function of two variables through a Barnes contour integral in the form

$$
\begin{aligned}
& G_{p, t, s, q, q}^{n, \nu_{1}, \nu_{2}, m_{1}, m_{2}}\left[\begin{array}{l|l}
\left(\epsilon_{p}\right) \\
x & \left(\gamma_{t}\right) ;\left(\gamma_{t}{ }^{\prime}\right) \\
y & \left(\delta_{s}\right) \\
\left(\beta_{q}\right) ;\left(\beta_{q}{ }^{\prime}\right)
\end{array}\right] \\
& =-\frac{1}{4 \pi^{2}} \int_{-i \infty}^{i \infty} \int_{-i \infty}^{i \infty} \Phi(\xi+\eta) \Psi(\xi, \eta) x^{\xi} y^{\eta} d \xi d \eta,
\end{aligned}
$$

where

$$
\begin{aligned}
& \Psi(\xi, \eta)=\Gamma\left[\begin{array}{l}
\left(\beta_{m_{1}}\right)-\xi,\left(\gamma_{\nu_{1}}\right)+\xi,\left(\beta_{m_{2}}^{\prime}\right)-\eta,\left(\gamma_{\nu_{2}}^{\prime}\right)+\eta ; \\
1-\left(\beta_{m_{1}+1, q}\right)+\xi, 1-\left(\gamma_{\nu_{1}+1, t}\right)-\xi, 1-\left(\beta_{m_{2}+1, q}^{\prime}\right)-\eta, 1-\left(\gamma_{\nu_{2}+1, t}\right)-\eta
\end{array}\right] ; \\
& \Phi(\xi+\eta)=\Gamma\left[\begin{array}{l}
1-\left(\epsilon_{n}\right)+\xi+\eta ; \\
\left(\epsilon_{n+1, p}\right)-\xi-\eta,\left(\delta_{s}\right)+\xi+\eta
\end{array}\right],
\end{aligned}
$$

and $0 \leqq m_{1} \leqq q, 0 \leqq m_{2} \leqq q, 0 \leqq \nu_{1} \leqq t, 0 \leqq \nu_{2} \leqq t, 0 \leqq n \leqq p$. The sequence of parameters $\left(\beta_{m_{1}}\right),\left(\beta_{m_{2}}^{\prime}\right),\left(\gamma_{\nu_{1}}\right),\left(\gamma_{\nu_{2}}^{\prime}\right)$ and $\left(\epsilon_{n}\right)$ are such that none of the poles of the integrand coincide. The paths of integration are indented, if necessary, in such a manner that all the poles of $\Gamma\left[\beta_{j}-\xi\right], j=1,2, \cdots, m_{1}$, and $\Gamma\left[\beta_{k}{ }^{\prime}-\eta\right], k=$ $1,2, \cdots, m_{2}$ lie to the right and those of $\Gamma\left[\gamma_{j}{ }^{\prime}+\eta\right], j=1,2, \cdots, \nu_{2} ; \Gamma\left[\gamma_{K}+\xi\right]$, $K=1,2, \cdots, \nu_{1}$ and $\Gamma\left[1-\epsilon_{j}+\xi+\eta\right], j=1,2, \cdots, n$ lie to the left of the imaginary axis.

The integral (19) converges if

$$
\begin{aligned}
& p+q+s+t<2\left(m_{1}+\nu_{1}+n\right), \\
& p+q+s+t<2\left(m_{2}+\nu_{2}+n\right),
\end{aligned}
$$

and

$$
\begin{aligned}
& |\arg x|<\pi\left(m_{1}+\nu_{1}+n-\frac{1}{2}[p+q+s+t]\right), \\
& |\arg y|<\pi\left(m_{2}+\nu_{2}+n-\frac{1}{2}[p+q+s+t]\right) .
\end{aligned}
$$

Now using the technique of Laplace transform and its inverse as used for the functions of one variable by Luke and Wimp [8] and by the author for obtaining general expansions of $G$-functions of Meijer [9], one can also write the extension to $G$-functions of all the results deduced in this paper. For instance, one can generalise (10) in the form 


$$
\begin{aligned}
& G_{\mu+p, \nu+q, \rho, \sigma+1}^{\mu, \nu+t, \nu+u, v, w}\left[\begin{array}{l}
\left(\alpha_{\mu+p}\right) \\
x \\
y \\
\left(\beta_{\nu+q}\right) ;\left(\beta_{\nu+q}^{\prime}\right) \\
\left(\gamma_{\rho}\right) \\
0,\left(\delta_{\sigma}\right) ; 0,\left(\delta_{\sigma}^{\prime}\right)
\end{array}\right] \\
& =\sum_{r=0}^{\infty} \frac{1}{[1]_{r}} G_{\mu+p, \nu+q+1, p+1, \sigma+1}^{\mu, \nu+t+1, \nu+u+1, v}\left[\begin{array}{l}
\left(\alpha_{\mu+p}\right) \\
a,\left(\beta_{\nu+q}\right) ; a,\left(\beta_{\nu+q}^{\prime}\right) \\
\left(\gamma_{\rho}\right), a \\
r,\left(\delta_{\sigma}\right) ; r,\left(\delta_{\sigma}{ }^{\prime}\right)
\end{array}\right] .
\end{aligned}
$$

The proof of this result is furnished by mathematical induction and for $t=u=$ $p=q=0, v=w=1$ this reduces to (10) on using the equation (iii) of $\S 3$ and (A) of $\$ 4$ of Agarwal [1].

Similarly all the other results can be extended to $G$-functions of two variables. These extended results were given earlier by Meijer [9], Luke and Wimp [8] and others.

Department of Mathematics

The University of Gorakhpur

Gorakhpur, India

1. R. P. Agarwal, "An extension of Meijer's G-functions," Proc. Nat. Inst. Sci. Sect. A, v. $32,1965$.

2. P. Appell \& J. Kampé de Fériet, Fonctions Hypergeometriques et Hyperspheriques, (iauthier-Villars, Paris, 1926.

3. J. L. Burchnali \& T. W. Chaundy, "Expansions of Appell's double hypergeometric functions," Quart. J. Math. Oxford Ser., v. 11, 1940, pp. 249-270. MR 2, 287.

4. J. L. Burchnall \& T. W. Chaundy, ibid., II, Quart. J. Math. Oxford Ser., v. 12, 1941, pp. 112-128. MR 3, 118

5. T. W. Chaundy, "Expansions of hypergeometric functions," Quart. J. Math. Oxford Ser., v. 13, 1942, pp. 159-171. MR 4, 197.

6. T. W. Chaundy, "On Clausen's hypergeometric identity," Quart. J. Math. Oxford Ser., 2, v. 9, 1958, pp. 265-274. MR 20 \$898.

7. J. L. FIELDS \& J. WIMP, "Expansions of hypergeometric functions in hypergeometric functions," Math. Comp., v. 15, 1961, pp. 390-395. MR 23 * A3289.

8. Y. L. LUKE \& JET WIMP, "Expansion formulas for generalized hypergeometric functions," Rend. Circ. Mat. Palermo (2), v. 11, 1962, pp. 351-366.

9. C. S. Meijer, a) "Expansion theorems for the G-functions," I, II, Nederl. Akad. Wetensch. Proc. Ser. A 55 = Indag. Math., v. 14, 1952, pp. 369-379, 483-487. MR 14, 469; MR 14,642 .

b) ibid., III, IV, V, Nederl. Akad. Wetensch. Proc. Ser. A $56=$ Indag. Math., v. 15, 1953, pp. 43-49, 187-193, 349-357. MR 14, 748; MR 14, 979; MR 15, 422.

c) ibid., VI, VII, VIII, Nederl. Akad. Wetensch. Proc. Ser. A $57=$ Indag. Math., v. 16, 1954, pp. 77-82, 83-91, 273-279. MR 15, 791; MR 15, 955.

d) ibid., IX, Nederl. Akad. Wetensch. Proc. Ser. A 58 = Indag. Math., v. 17, pp. 243-251. MR 16, 1106.

10. J. D. Niblett, "Some hypergeometric identities," Pacific J. Math., v. 2, 1952, pp. 219-225. MR 13, 940.

11. F. M. RAGAB, "Expansions of Kampé de Fériet's double hypergeometric functions of higher order," J. Reine. Angew. Math., v. 212, 1963, pp. 113-119. MR 27 *352.

12. A. Verma, "A class of expansions of $G$-functions and Laplace transform," Math. Comp., v. 19,1965 , pp. $661-664$. 